\title{
Stripes and Pairing in High Temperature Superconductors*
}

\author{
AndRZEJ M. Oleś ${ }^{a, b}$ \\ ${ }^{a}$ Marian Smoluchowski Institute of Physics, Jagellonian University, Reymonta 4, PL-30059 Kraków, Poland \\ ${ }^{b}$ Max-Planck-Institut für Festkörperforschung, Heisenbergstrasse 1, D-70569 Stuttgart, Germany \\ We review briefly several approaches used to investigate the stability of stripe phases in high temperature \\ superconductors, where charge inhomogeneities arise from competing kinetic and magnetic energies. The mecha- \\ nism of stripe formation, their consequences for the normal state and enhancement of pairing interaction triggered \\ by charge inhomogeneities are briefly summarized. Finally, we demonstrate that orbital degeneracy $(i)$ leads to a \\ more subtle mechanism of stripe formation, and (ii) plays an important role and determines the symmetry of the \\ superconducting state in pnictides.
}

PACS: 75.10.Jm, 75.30.Et, 03.67.Mn, 61.50.Ks

\section{Models for $\mathrm{CuO}_{2}$ planes}

There are several round anniversaries this year related to the research on superconductivity - not only 100 years since the discovery of this phenomenon in mercury and 25 years since the discovery of high temperature superconductors (HTSC). This latter discovery triggered increased interest in the properties of strongly correlated electrons on a two-dimensional (2D) square lattice. The simplest model to describe the behavior of correlated electrons in HTSC is the Hubbard model, or the effective $t-J$ model derived from it 35 years ago [1]. This model was ready at the moment when the HTSC were discovered and could be used to investigate their behavior [2].

At present we know that the Hubbard (or $t-J$ ) model provides the simplest description of the electronic states in $\mathrm{CuO}_{2}$ planes, being a common structural element of HTSC. The minimal realistic model for $\mathrm{CuO}_{2}$ planes is the so-called three-band $d-p$ (charge-transfer) model, introduced simultaneously by a few theoretical groups [3-5] - it contains hybridized $\mathrm{Cu}\left(3 d_{x 2-y 2}\right)$ and $\mathrm{O}\left(2 p_{\sigma}\right)$ orbitals, with Coulomb on-site interactions $U_{d}$ and $U_{p}$ between two electrons with opposite spins, and the intersite Coulomb repulsion $U_{d p}$. At this early stage of the theory of superconductivity in HTSC it was important to establish how strongly the $3 d$ electrons are correlated. This was investigated using a variational local ansatz to the three-band model [4]. This approach was also employed at that time to establish universal features concerning the correlation strength for $\sigma, \pi$ and aromatic molecular bonds [6]. One finds that all these bonds are weaker correlated than hybridized $d-p$ electrons, both in $\mathrm{CuO}_{2}$ planes [4] and in $\mathrm{CuO}_{3}$ chains [7] found in $\mathrm{YBa}_{2} \mathrm{Cu}_{3} \mathrm{O}_{7}$

\footnotetext{
* This article is dedicated to Professor Karol I. Wysokiński on the occasion of his 60th birthday.
}

superconductor. This implies that charge fluctuations are almost entirely suppressed and only certain delocalization of holes centered at $\mathrm{Cu}$ sites over the neighboring $\mathrm{O}\left(2 p_{\sigma}\right)$ orbitals takes place due to $d-p$ hybridization $t_{p d}$ [4]. On the other hand, the effective superexchange interaction along the $\mathrm{Cu}-\mathrm{O}-\mathrm{Cu}$ bonds between the $S=1 / 2$ spins at $\mathrm{Cu}$ ions leads to an antiferromagnetic (AF) order [8]. This result served as a justification to use the effective Hubbard model instead of the more complete three-band model.

In HTSC it is more convenient to introduce the hole notation as the reference state in $\mathrm{La}_{2} \mathrm{CuO}_{4}$ contains one hole at each $\mathrm{Cu}^{2+}$ ion in the $d^{9}$ configuration. Doping in $\mathrm{La}_{2-x} \mathrm{Sr}_{x} \mathrm{CuO}_{4}$ or in $\mathrm{YBa}_{2} \mathrm{Cu}_{3} \mathrm{O}_{6+x}$ (where the $\mathrm{CuO}_{3}$ chains gradually form) generates holes in oxygen orbitals within $\mathrm{CuO}_{2}$ planes and leads to formation of local Zhang-Rice singlets [9]. This concept is crucial as a doped hole occupies not a $\mathrm{Cu}\left(d_{x^{2}-y^{2}}\right)$ orbital but a linear combination of $p_{\sigma}$ orbitals around a hole with $x^{2}-y^{2}$ symmetry and forms a singlet together with the hole at the $\mathrm{Cu}$ ion. Therefore, adding a hole into a $\mathrm{CuO}_{2}$ plane may be viewed as a removal of one $\mathrm{Cu}$ spin in the effective Hubbard model that describes the Mott insulator,

$$
\hat{H}=-\sum_{i j, \sigma} t_{i j} \hat{a}_{i \sigma}^{\dagger} \hat{a}_{j \sigma}+U \sum_{i} \hat{n}_{i \uparrow} \hat{n}_{i \downarrow}
$$

where $\hat{a}_{i \sigma}^{\dagger}$ is a hole creation operator in an $|i \sigma\rangle$ state and $\hat{n}_{i \sigma}=\hat{a}_{i \sigma}^{\dagger} \hat{a}_{i \sigma}$. Here $t_{i j} \equiv t$ or $t_{i j} \equiv t^{\prime}$ are hopping elements between the nearest neighbor (NN) or next-nearest neighbor (NNN) $\mathrm{Cu}$ ions, and $U$ is an effective on-site Coulomb parameter. The latter stands for the chargetransfer gap $\Delta$ in the electronic structure of the threeband $d-p$ model and is much lower than $U_{d} \simeq 10 \mathrm{eV}$. The parameters in Eq. (1) can be derived from the electronic structure calculations [10] (see also [11]), and one finds $t \equiv t_{p d}^{2} / \Delta \simeq 0.4 \mathrm{eV}$ and $U \simeq 4 \mathrm{eV}$.

The three-band model with the Zhang-Rice singlets 
and the above Hubbard model (1) are two independent routes toward the effective $t-J$ model, derived using perturbation theory in Cracow 35 years ago (and published one year later [1]) — another round anniversary — this model describes the electronic states in cuprates [2]. A properly chosen canonical transformation leads from the full Hilbert space to an effective low-energy Hamiltonian acting in the restricted space. The model consists of the kinetic energy $\propto t$ (here we assume $t^{\prime}=0$ ) and the AF superexchange $\propto J \equiv 4 t^{2} / U$ between $S=1 / 2$ spins:

$$
\hat{\mathcal{H}}_{t-J}=-\sum_{i j, \sigma} t_{i j} \hat{\tilde{a}}_{i \sigma}^{\dagger} \hat{\tilde{a}}_{j \sigma}+J \sum_{\langle i j\rangle}\left(\hat{\boldsymbol{S}}_{i} \cdot \hat{\boldsymbol{S}}_{j}-\frac{1}{4} \hat{\tilde{n}}_{i} \hat{\tilde{n}}_{j}\right) .
$$

The operators $\hat{\tilde{a}}_{i \sigma}^{\dagger}=\hat{a}_{i \sigma}^{\dagger}\left(1-\hat{n}_{i \bar{\sigma}}\right)(\bar{\sigma}=-\sigma)$ are projected fermion operators and act in the restricted space. The above $t-J$ model (or $t-t^{\prime}-J$ model when $t^{\prime} \neq 0$ ) follows also directly from the three-band $d-p$ model - the superexchange in cuprates includes both the Anderson and charge-transfer excitations [8] and stabilizes 2D AF order at $x=0$ [12]. The value of $J \simeq 0.13 \mathrm{eV}$ is either deduced from the magnetic experiments, or derived from the parameters of the charge-transfer model [10].

The first intriguing question concerning hole doping is whether a hole doped to the AF state may propagate coherently. Naively one might argue that a hole creates defects on its way, so it would need to make a hopping along a closed loop to annihilate these defects and to propagate in the square lattice [13]. Actually, this is the only possible process to delocalize the hole in the Ising model, see also Section 4. But when a hole is doped into a Heisenberg antiferromagnet the quantum fluctuations of the AF background repair the defects created by the hole, resulting in the propagation of a hole in form of a quasiparticle (QP) with dispersion on the energy scale of $J[14]$. This concept was confirmed by angle resolved photoemission (ARPES) experiments in cuprates [15]. Detailed comparison between the experimental data of ARPES experiments and the outcome of the theoretical calculations performed using the self-consistent Born approximation (SCBA) [14] were presented by several groups. One of the highlights is the theoretical explanation of high quality ARPES data obtained for $\mathrm{Sr}_{2} \mathrm{CuO}_{2} \mathrm{Cl}_{2}$ which are reproduced by the SCBA calculations performed for $t^{\prime}=-0.3 t$ [16]. This demonstrates that the $t-t^{\prime}-J$ model is the right effective model for the HTSC.

The remaining of this paper is organized as follows. We analyze the origin and physical properties of stripe phases in Section 2, and show in Section 3 that they may enhance the pairing interaction. Stripe phases may also form in systems with alternating orbital order but their origin is more subtle as shown in Section 4. There we also point out that Hund's exchange and orbital physics play an important role in pnictides. Short summary is presented in Section 5. Original graphical material on the stripe phases may be found in the cited literature.

\section{Microscopic origin of stripe phases}

Doping of $\mathrm{CuO}_{2}$ planes weakens AF correlations which become short-range but survive up to the overdoped regime $(x \simeq 0.2)[12]$. While $\mathrm{AF}$ and superconducting (SC) states exist in distinct regimes, there are several possibilities how the phase diagram could look like when doping increases [17]. In fact, the doped holes selforganize in form of phases with charge and magnetization density modulation, called stripe phases [18]. Such structures, with the charge density varying twice faster than the magnetization density in real space [19, 20], were first discovered in the theory as an instability of doped antiferromagnets. Only a few years later their existence in cuprates was confirmed by neutron experiments [21].

The first question concerning stripe phases is whether they are as solitonic defects in the AF structure, i.e., separating different AF domains, or they form as polarons in a single AF domain. Although naively one could argue that usually polarons optimize better the kinetic energy, the answer is more subtle. The simplest stability estimate is obtained by considering a three-site cluster filled by two electrons and centered at the domain wall [22]. Due to self-organization at large $U \gg t$, the electrons are confined within the cluster and their configurations with either identical or opposite spins correspond to: $(i)$ polaronic, and $(i i)$ solitonic unit, with energies $E_{P}$ and $E_{S}$. One finds $E_{P}=-\sqrt{2} t$ and $E_{S}=E_{P}-4 t^{2} / U$, i.e., the solitonic energy is lower by the superexchange energy $J$ due to the three-site effective hopping processes [22]. This simple argument explains the experimental finding [21] that charge walls are nonmagnetic and separate domains with different phase of the AF order parameter.

First quantitative results for the stripe phases were obtained within the Hartree-Fock (HF) approximation [19, 20], and then refined using variational wave functions [23] and rotationally invariant version of the slave-boson approach formulated as renomalized mean field theory (RMFT) with Gutzwiller renormalization [24]. The HF studies gave remarkably robust insulating vertical (or horizontal) (01) stripe structures with the observed filling of one doped hole per two domain wall sites [21], and with rather large charge density modulation between the centers of AF domains and the domain walls [20]. The smallest charge unit cell found contains four atoms which explains that the stripes melt in the overdoped regime $x>0.19$. Evolution of the electronic structure described within the HF studies shows systematic changes of the Fermi surface, depending on the ratio of the charge incommensurability and hole doping $x[25]$.

In the HF approach the stripes are stabilized by certain additional (spin or charge) density modulation along the domain walls [20], so one expects major changes when electron correlations are included. Indeed, the modulation of charge and magnetization density was reduced from the HF values within the RMFT for the 2D Hubbard model [24]. This approach allowed one to treat strong electron correlations in stripe phases with large unit cells 
relevant in the low doping regime, and gave stable stripes in the thermodynamic limit. It also helped to resolve the longstanding controversy concerning the role played by the kinetic energy in the stripe phases. While the transverse hopping across the domain walls yields the largest kinetic energy gain in case of insulating stripes with one hole per site, the holes propagating along the domain walls stabilize instead metallic vertical (01) stripes, with one hole per two sites in the cuprates.

Although the long-range Coulomb interaction might help to stabilize the stripe order further, the great success of the real-space dynamical mean field theory (DMFT) studies for the 2D Hubbard model was the proof that the correct treatment of the on-site interaction alone suffices to stabilize experimentally observed metallic stripes in a rather broad doping range $0.03<x<0.2$ [26]. These calculations reproduced also the observed crossover from diagonal (11) to vertical (01) site-centered stripes at doping $x \simeq 0.05$ [27]. In addition, also the doping dependence of the size of magnetic domains and the shift of the chemical potential, $\Delta \mu \propto-x^{2}$, were found to be in quantitative agreement with the experimental results for $\mathrm{La}_{2-x} \mathrm{Sr}_{x} \mathrm{CuO}_{4}$. In this way the paradigm of insulating stripe phases was abolished - the chemical potential was decreasing with doping within the metallic phase.

The spectral functions obtained within the DMFT [26] show a coexistence of the incoherent states in the lower Hubbard band and coherent QP states close to the Fermi energy. The main features of the spectra are: a flat part of the QP band near the $X=(\pi, 0)$ point, and gaps for charge excitations at the $Y=(0, \pi)$ and $S=(\pi / 2, \pi / 2)$ points in the low-doping regime $x<1 / 8$. These gaps are gradually filled and close up under increasing doping, in agreement with the experimental ARPES data for $\mathrm{La}_{2-x} \mathrm{Sr}_{x} \mathrm{CuO}_{4}$ [28]. In a range of low temperature the obtained spectra have a distinct QP peak at the $X$ point, present just below the Fermi energy $\mu$, a charge gap, and a distinct QP at the $S$ point [26]. At increasing temperature the spectral function $A(\boldsymbol{k}, \omega)$ gradually changes, indicating the melting of stripe order irrespective of the choice of the NNN hopping $t^{\prime}$ [29].

These calculations demonstrated [26] the importance of dynamical correlations which strongly screen the local potentials resulting from the on-site Coulomb interaction $U$ and lead thus to drastic changes in the distribution of spectral weight with respect to the HF picture. It was also shown [26] that the melting of stripe order is influenced by the NNN hopping element $t^{\prime}$, which also explains the observed difference in the spectral properties between $\mathrm{Bi}_{2} \mathrm{Sr}_{2} \mathrm{CaCu}_{2} \mathrm{O}_{8+y}$ [30] and $\mathrm{La}_{2-x} \mathrm{Sr}_{x} \mathrm{CuO}_{4}$ [28]. At the same time, $t^{\prime}$ can tip the energy balance between the filled diagonal and half-filled vertical stripes [24], which might explain a change in the spatial orientation of stripes observed in the HTSC at doping $x \simeq 1 / 16$.

\section{Charge inhomogeneities and pairing}

After understanding the mechanism of stripe formation in cuprates, a natural question to ask concerns their role in the phenomenon of superconductivity. This subject is relatively new and currently under investigation by several groups. While low temperature properties of the SC state cannot be explained within the original resonating valence-bond (RVB) framework [2, 31], evidence accumulates that $\mathrm{SC}$ correlations coexist in these systems with charge inhomogeneities. Microscopic evidence of inhomogeneities given by scanning tunnelling microscopy (STM) [32] motivated several recent studies in the theory. The simplest mean-field Hamiltonian for a singlet superconductor with disorder in pairing interaction gives indeed a distribution of gap values in the local density of states (DOS) found by solving self-consistently Bogoliubov-de Gennes equations [33]. The above disorder follows here from dopant interstitial $\mathrm{O}$ atoms which modify the local electronic structure [34].

Pioneering work in this respect was presented by Maśka et al. [35] who provided a beautiful explanation of the origin of the experimentally observed positive correlation between the magnitude of the SC gap and positions of dopant oxygens in Bi-based superconductors. Their approach demonstrated that charge inhomogeneities, caused by spatial variation of the atomic levels, are responsible for local enhancement of the pairing interaction $J$ in the effective $t-J$ model. However, in the more complete three-band model one finds that the sign of the correction to $J$ depends on the potentials on nearby sites induced by the dopant impurity, suggesting important corrections beyond the one-band model [36].

Coexisting charge modulation and unidirectional $d$ wave SC domains were found using the $t-J$ model at $x=1 / 8$ doping [37]. In this study half-filled charge domains separated by four lattice spacings were obtained along one of the crystal axes leading to modulated superconductivity with out-of-phase $d$-wave order parameters in neighboring domains. Both the RMFT and variational Monte Carlo calculations yield that the unidirectionally modulated superconducting phases are energetically remarkably close to the uniform RVB phase, so they could easily be stabilized by impurities or other effects going beyond the $t-J$ model [38]. These studies were also extended to the pyrochlore lattice, where the phase diagram includes superconductivity coexisting with the underlying valence-bond solid order [39]. This interesting work demonstrates that the interplay between electron correlation and geometrical frustration can stabilize novel states of matter exhibiting microscopic coexistence of superconductivity and spin dimer order.

Exact diagonalization of finite clusters of size $N$ (with periodic boundary conditions) is an ideal unbiased method to investigate pairing in models of interacting electrons [40]. This method was used recently to investigate pairing in inhomogeneous $4 \times 4$ clusters described by the Hubbard model (1) with inequivalent hopping integrals along two directions in a $2 \mathrm{D}$ plane, $t_{a}$ and $t_{b}<t_{a}$, in two patterns: checkerboard and stripe phase [41]. The pair binding energy at doping $x=M / N$, 


$$
\Delta_{\mathrm{B}}(x)=2 E_{0}(M)-\left(E_{0}(M+1)+E_{0}(M-1)\right) .
$$

is defined by comparing the ground state energies $E_{0}(M)$ obtained for systems with separated holes and a hole pair for electron number $M$ [40]. The pair-binding energy $\Delta_{\mathrm{B}}(x)>0$ is found in both considered structures with purely repulsive interactions. At $x=1 / 16$ it has the largest value in the intermediate regime of $t_{b} \simeq 0.6 t_{a}$ and $U \simeq 8 t_{a}$ for the checkerboard lattice, but also for the stripe lattice this energy has a maximum close to $U=12 t_{a}[41]$.

Further evidence that charge inhomogeneities enhance superconductivity has accumulated from cluster dynamical studies for the Hubbard model [42]. The electronic properties of multilayers with combinations of underdoped and overdoped layers were investigated using cluster DMFT superlattices. It has been found that the $\mathrm{SC}$ order parameter is enhanced by the proximity of the strong pairing scale originating from the underdoped layers and can even exceed the maximum value in uniform systems. In quantum Monte Carlo simulations stripelike charge-density wave modulation was imposed by a periodic potential with modulation strength $V_{0}$ and the pairing correlations and critical temperature were determined from the Bethe-Salpeter equation in the particleparticle channel [42]. The optimal superconductivity is then obtained for a moderate modulation strength due to a delicate balance between the modulation enhanced pairing and suppression of the pure particle-particle excitations by a modulation reduction of the QP weight.

Recently stripe phases were also investigated in the $2 \mathrm{D}$ $t-J$ model by means of infinite projected entangled-pair states [43]. The states with stripe order were found to have a lower variational energy than uniform phases, and the stripes support $d$-wave pairing. For a fixed unit-cell size the energy per hole is minimized for a hole density of $1 / 2$ hole per unit length of a stripe. These results support earlier findings obtained within the RMFT [24] and the DMFT [26]. In addition, one finds that the pairing amplitude is largest at the atoms with enhanced hole density and suppressed magnetization, and the mean pairing amplitude has a characteristic maximum as a function of hole density per unit length of stripe [43]. Mean field pairing theory for the charge stripe phase of HTSC was just formulated [44] - the model describes the most prominent properties of the stripe phase remarkably well and predicts a pair density wave with spatial modulation of the pairing amplitude in the striped structure.

Experimental evidence accumulates that stripe order coexists with the SC order. In fact, as the most likely interpretation of the complete collection of results obtained for magnetic susceptibility, thermal conductivity, specific heat, resistivity and thermopower for $\mathrm{La}_{1.875} \mathrm{Ba}_{0.125} \mathrm{CuO}_{4}$, appearance of unusual 2D SC correlations together with the onset of spin-stripe correlations was suggested [45]. This suggestion seems to reflect the real situation as confirmed by extensive studies of $\mathrm{La}_{2-x} \mathrm{Ba}_{x} \mathrm{CuO}_{4}$ compounds in the broad range of doping, $0.095<x<0.155$ [46]. Stripe incommensurability increases here with $x$ in a smooth way, unlike in the earlier data for $\mathrm{La}_{2-x} \mathrm{Sr}_{x} \mathrm{CuO}_{4}$ [27]. In the entire range of doping $x$ the charge order appears at a higher temperature $T_{\mathrm{CO}}$ than the onset of spin order at $T_{\mathrm{SO}}$. Truly static spin order sets in below the charge order and coincides with the first appearance of in-plane SC correlations at temperatures significantly above the SC transition in the bulk. They also presented an interesting phase diagram [46] and argued that charge order is the dominant order that is compatible with $\mathrm{SC}$ pairing but competes with SC phase coherence.

Local enhancement of pairing mechanism may occur even in absence of stripe order, as long as charge inhomogeneities are induced by structure charges. For instance, apical oxygens may affect the strength of the pairing potential within $\mathrm{CuO}_{2}$ layers [47]. In fact, the superexchange is very sensitive to the covalency which changes depending on the position of apical oxygens. This is due to high polarizability of $\mathrm{O}^{2-}$ anion which has the effect of screening and reduces the Coulomb interaction $U$ [48]. Hole density inhomogeneities that exist in $\mathrm{HgBa}_{2} \mathrm{CuO}_{4+\delta}$ and $\mathrm{Bi}_{2} \mathrm{Sr}_{2} \mathrm{CaCu}_{2} \mathrm{O}_{8+\delta}$ are expected to play a similar role and enhance the pairing locally [49].

\section{Orbital degeneracy and pnictides}

In contrast to cuprates, iron-based superconductors contain several partly filled bands and orbital degeneracy plays a role. Local electron interactions are then described by degenerate Hubbard model with two parameters: intraorbital Coulomb element $U$ and Hund's exchange $J_{H}$ [50]. When the spin-orbital superexchange model is derived for such a Mott insulator at $t \ll U$, the multiplet structure of excited states decides about different spin-orbital terms that depend on $J_{H}$ and determine both the magnetic properties and the optical spectral weights [51]. For instance, the superexchange in manganites explains the observed spin and orbital order in $\mathrm{LaMnO}_{3}$ [52], while the relevant spin-orbital $t-J$ model for $e_{g}$ electrons gives a correct description of the metallic FM phase including the origin of spin excitations [53], and was also used to investigate the evolution of magnetic order with increasing doping in doped momolayer and bilayer manganites [54].

Stripe phases appear also in doped transition metal oxides with active orbital degrees of freedom and some examples were given in Refs. [11, 17]. Here we address briefly only the simplest case of stripe order in doped $\mathrm{La}_{2-x} \mathrm{Sr}_{x} \mathrm{NiO}_{4}$ with AF order found about the same time as in cuprates [55]. In contrast to cuprates, however, the stripes in $\mathrm{La}_{5 / 3} \mathrm{Sr}_{1 / 3} \mathrm{NiO}_{4}$ are diagonal and contain one hole per unit cell [56]. This difference follows from orbital degeneracy and realistic $e_{g}$ hopping that does not conserve the orbital flavour [57]. Electronic structure calculations suggest that a subtle interplay between the charge and spin order and octahedral distortions is essential for the formation of an insulating state [58]. Indeed, both local electron correlations and the Jahn-Teller interactions with lattice distortions play a role in reproducing 
experimentally observed stripes at $x=1 / 3$ doping and the checkerboard structure at $x=1 / 2$ [59].

In systems with active $t_{2 g}$ degrees of freedom and FM spin order, alternating orbital order follows from Isinglike superexchange and stable stripe phases are different. The orbital $t-J$ model that follows in this case from the degenerate Hubbard model on the square lattice [60] is applicable either to transition metal oxides with active $t_{2 g}$ orbitals filled by one electron at each site (with $\{y z, z x\}$ doublet), or to cold-atom systems with active $p$ orbitals. Although the Ising superexchange suggests that the holes would be confined, the system may self-organize at finite doping in a form of stripe phase [61]. In cuprates the presence of quantum spin fluctuations favors stripes in form of ladders with dominating singlet correlations on the rungs [62]. Here quantum spin fluctuations are absent and orbitals reorient into ferro-orbitally ordered domain walls that allow for deconfined motion of holes along them [63]. These solitonic stripes are more stable than polaronic stripes, but the phase change of the staggered order by $\pi$ plays a minor role in orbitally ordered systems and both types of stripes might be expected at finite temperature. The ferro-orbital order is induced by the kinetic energy of doped holes and occurs here by the same mechanism as in $e_{g}$ orbital systems [64].

Coming to iron-based pnictides, several families of them exist (for more details see a recent review [65]), and it is a challenge for the theory to explain the origin of the SC pairing and its symmetry. It was initially speculated that the pairing in these new superconductors is related to that of the cuprates, but de facto the situation is by far more complex. First, similar to cuprates, AF interactions decide about magnetic order in pnictides in the vicinity of $\mathrm{SC}$ states [66]. One finds a spin-density wave or a $C$-type $\mathrm{AF}(C-\mathrm{AF})$ phase, sometimes incorrectly called "a stripe phase" (this phase occurs here without doping). Second, the electronic structure of pnictides was investigated in detail and it was shown that all $3 d$ states of Fe ions are partly filled [67] and a priori play an important role in the electronic instabilities. Electronic structure calculations predict the magnetic order with large magnetic moments but the observed moments are small $\approx 0.2 \mu_{\mathrm{B}}$. This problem can be resolved [67] by reducing the Stoner parameter $I$ which contains two systematic errors when derived from local density approximation (LDA) [68]. In fact, the LDA + DMFT calculation demonstrate that [69]: (i) Hund's exchange $J_{H}$ is important and decides about the actual values of the resistivity and specific heat in pnictides, and $(i i)$ the magnetic moment in the $C-\mathrm{AF}$ phase is much reduced from the LDA value.

It has been established by now that the superconductivity in layered iron-based materials occurs by unconventional mechanism [70]. Impurities play a similar role as in cuprates and locally modify pairing conditions in pnictide superconductors [71]. The structure of the Fermi surface is rich and several orbital states could be involved in the pairing [67]. Therefore, next to AF interactions, the orbital degeneracy plays here a very important role and the microscopic models have to treat explicitly at least two orbitals per site. Interorbital pairing interactions arise in such a model and stabilize $s_{ \pm}$-wave symmetry of the superconducting order parameter [71]. However, the symmetry of the superconducting state in pnictides is still controversial and currently under investigation.

The two-orbital model was suggested shortly after these systems were discovered as a generic model to investigate both the magnetic order and the pairing [72]. This model includes $t_{2 g}$ partly filled orbitals $\{x z, y z\}$ and the electronic structure consists of a wide band and a narrow band [73]. Keeping only these orbitals is reasonable knowing that $x z$ and $y z$ orbitals provide the largest contribution to the pnictides' Fermi surface [67]. Superexchange interactions obtained in the spin-orbital model are here frustrated as both nearest neighbor $\left(J_{\mathrm{NN}}\right)$ and next-nearest neighbor $\left(J_{\mathrm{NNN}}\right)$ exchange is AF [74].

Recent Lanczos diagonalization studies of the $t-U-J$ model using a small $\sqrt{8} \times \sqrt{8}$ cluster and Eq. (3) with $M=18$ to determine the pairing energy showed [75] that Hund's exchange $J_{H}$ is crucial also here, together with the AF frustrated superexchange. One finds that the $A_{1 g}$ and $B_{2 g}$ pairing symmetries compete with each other in the realistic parameter regime. Quasinodal $A_{1 g}$ states are stabilized for physical values of $J_{H} / U$ and for sufficiently large $U$, in agreement with the RPA results [76], but $B_{1 g}$ pairing symmetry is also energetically close and could be stabilized by other weak interactions. The two-orbital model is oversimplified but its results [75] agree qualitatively with those obtained in the mean-field approximation for the three-orbital model [77]. Here the pairing instabilities also highlight the importance of Hund's exchange in pnictides.

\section{Summary and conclusions}

Summarizing, stripe phases arise from a competition between the superexchange energy of localized spins and the kinetic energy of doped holes as a joint instability toward coexisting spin and charge modulated order. Recent results suggest that the stripe phases may coexist with superconductivity. In fact, the pairing interactions in cuprates are enhanced by charge inhomogeneities that are generated by doping. Further theoretical progress in the understanding of stripe phases and their consequences for the pairing requires sophisticated self-consistent cluster calculations for stripe phases.

In pnictide superconductors necessary ingredients of the theory are orbital degeneracy and Hund's exchange. This makes the theoretical studies of the pairing mechanism in pnictides more demanding but also here the AF superexchange interactions are crucial for the pairing mechanism.

\section{Acknowledgments}

It is a great pleasure to thank for so pleasant collaboration and for numerous insightful discussions - I thank 
in particular Raymond Frésard, Piotr Wróbel and Jan Zaanen, as well as M. Daghofer, E. Dagotto, D. Góra, M. Fleck, A.I. Lichtenstein, A. Moreo, D. Poilblanc, M. Raczkowski, K. Rościszewski and G.A. Sawatzky. We acknowledge support by the Polish National Science Center (NCN) under Project No. N202 069639.

\section{References}

[1] K.A. Chao, J. Spałek, A.M. Oleś, J. Phys. C 10, L271 (1977); K.A. Chao, J. Spałek, A.M. Oleś, Phys. Rev. B 18, 3453 (1978).

[2] M. Ogata, H. Fukuyama, Rep. Prog. Phys. 71, 036501 (2008); E. Arrigoni, M. Aichhorn, M. Daghofer, W. Hanke, New J. Phys. 11, 055066 (2009).

[3] V.J. Emery, Phys. Rev. Lett. 58, 2794 (1987).

[4] A.M. Oleś, J. Zaanen, P. Fulde, Physica BEC 148, 260 (1987).

[5] C.M. Varma, S. Schmitt-Rink, E. Abrahams, Solid State Commun. 62, 681 (1987).

[6] A.M. Oleś, F. Pfirsch, P. Fulde, M.C. Böhm, J. Chem. Phys. 85, 5183 (1986); A.M. Oleś, F. Pfirsch, P. Fulde, M.C. Böhm, Z. Phys. B 66, 359 (1987).

[7] A.M. Oleś, W. Grzelka, Phys. Rev. B 44, 9531 (1991).

[8] J. Zaanen, A.M. Oleś, Phys. Rev. B 37, 9423 (1988).

[9] F.C. Zhang, T.M. Rice, Phys. Rev. B 37, 3759 (1988).

[10] J.B. Grant, A.K. McMahan, Phys. Rev. B 46, 8440 (1992).

[11] A.M. Oleś, Acta Phys. Pol. A 118, 212 (2010).

[12] M.A. Kastner, R.J. Birgeneau, G. Shirane, Y. Endoh, Rev. Mod. Phys. 70, 897 (1998).

[13] S.A. Trugman, Phys. Rev. B 37, 1597 (1988).

[14] G. Martínez, P. Horsch, Phys. Rev. B 44, 317 (1991).

[15] A. Damascelli, Z. Hussain, Z.-X. Shen, Rev. Mod. Phys. 75, 473 (2003).

[16] J. Bała, A.M. Oleś, J. Zaanen, Phys. Rev. B 52, 4597 (1995).

[17] E. Dagotto, New J. Phys. 7, 67 (2005).

[18] S. Sachdev, Rev. Mod. Phys. 75, 913 (2003); M. Raczkowski, A.M. Oleś, R. Frésard, Low Temp. Phys. 32, 305 (2006); M. Vojta, Adv. Phys. 58, 699 (2009).

[19] J. Zaanen, O. Gunnarsson, Phys. Rev. B 40, 7391 (1989); D. Poilblanc, T.M. Rice, Phys. Rev. B $\mathbf{3 9}$ 9749 (1989); B. Normand, A.P. Kampf, Phys. Rev. B 64, 024521 (2001).

[20] J. Zaanen, A.M. Oleś, Ann. Phys. 5 (508), 224 (1996).

[21] J.M. Tranquada, B.J. Sternlieb, J.D. Axe, Y. Nakamura, S. Uchida, Nature (London) 375, 561 (1995); M. Fujita, H. Goka, K. Yamada, J.M. Tranquada, L.P. Regnault, Phys. Rev. B 70, 104517 (2004).

[22] A.M. Oleś, Acta Phys. Pol. B 31, 2963 (2000).

[23] D. Góra, K. Rościszewski, A.M. Oleś, Phys. Rev. B 60, 7429 (1999).

[24] M. Raczkowski, R. Frésard, A.M. Oleś, Phys. Rev. B 73, 174525 (2006); M. Raczkowski, R. Frésard, A.M. Oleś, Europhys. Lett. 76, 128 (2006).

[25] M. Ichioka, K. Machida, J. Phys. Soc. Jpn. 68, 4020 (1999).
[26] M. Fleck, A.I. Lichtenstein, E. Pavarini, A.M. Oleś, Phys. Rev. Lett. 84, 4962 (2000); M. Fleck, A.I. Lichtenstein, A.M. Oleś, Phys. Rev. B 64, 134528 (2001).

[27] K. Yamada, C.H. Lee, K. Kurahashi, J. Wada, S. Wakimoto, S. Ueki, H. Kimura, Y. Endoh, S. Hosoya, G. Shirane, R.J. Birgeneau, M. Greven, M.A. Kastner, Y.J. Kim, Phys. Rev. B 57, 6165 (1998).

[28] A. Ino, C. Kim, M. Nakamura, T. Yosida, T. Mizokawa, Z.X. Shen, A. Fujimori, T. Kakeshita, H. Eisaki, S. Uchida, Phys. Rev. B 62, 4137 (2000).

[29] M. Raczkowski, F.F. Assaad, Phys. Rev. B 82, 233101 (2010).

[30] A. Ino, C. Kim, T. Mizokawa, Z.-X. Shen, A. Fujimori, M. Takaba, K. Tamasaku, H. Eisaki, S. Uchida, J. Phys. Soc. Jpn. 68, 1496 (1999).

[31] P.W. Anderson, P.A. Lee, M. Randeria, T.M. Rice, N. Trivedi, F.C. Zhang, J. Phys.: Condens. Matter 16 R755 (2004); J.Y. Gan, F.C. Zhang, Z.B. Su, Phys. Rev. B 71, 014508 (2005).

[32] T. Cren, D. Roditchev, W. Sacks, J. Klein, Europhys. Lett. 54, 84 (2001).

[33] T.S. Nunner, B.M. Andersen, A. Melikyan, P.J. Hirschfeld, Phys. Rev. Lett. 95, 177003 (2005).

[34] Y. He, T.S. Nunner, P.J. Hirschfeld, H.-P. Cheng, Phys. Rev. Lett. 96, 197002 (2006).

[35] M.M. Maśka, Ż. Śledź, K. Czajka, M. Mierzejewski, Phys. Rev. Lett. 99147006 (2007).

[36] K. Foyevtsova, R. Valentí, P.J. Hirschfeld, Phys. Rev. B 79, 144424 (2009).

[37] M. Raczkowski, M. Capello, D. Poilblanc, R. Frésard, A.M. Oleś, Phys. Rev. B 76, 140505 (2007).

[38] M. Capello, M. Raczkowski, D. Poilblanc, Phys. Rev. B 77, 224502 (2007); M. Raczkowski, Acta Phys. Pol. A 114, 107 (2008)

[39] M. Raczkowski, D. Poilblanc, Phys. Rev. Lett. 103 , 027001 (2009).

[40] J. Bonča, P. Prelovšek, I. Sega, Phys. Rev. B 39, 7074 (1989).

[41] W.-F. Tsai, H. Yao, A. Läuchli, S.A. Kivelson, Phys. Rev. B 77, 214502 (2008).

[42] S. Okamoto, T.A. Maier, Phys. Rev. Lett. 101 , 156401 (2008); T.A. Maier, G. Alvarez, M. Summers, T.C. Schulthess, Phys. Rev. Lett. 104, 247001 (2010).

[43] P. Corboz, S.R. White, G. Vidal, M. Troyer, Phys. Rev. B 84, 041108 (2011).

[44] F. Loder, S. Graser, A.P. Kampf, T. Kopp, Phys. Rev. Lett. 107, 187001 (2011).

[45] J.M. Tranquada, G.D. Gu, M. Hücker, Q. Jie, H.J. Kang, R. Klingeler, Q. Li, N. Tristan, J.S. Wen, G.Y. Xu, Z.J. Xu, J. Zhou, M.V. Zimmermann, Phys. Rev. B 78, 174529 (2008).

[46] M. Hücker, M.v. Zimmermann, G.D. Gu, Z.J. Xu, J.S. Wen, G. Xu, H.J. Kang, A. Zheludev, J.M. Tranquada, Phys. Rev. B 83, 104506 (2011).

[47] M. Mori, G. Khaliullin, T. Tohyama, S. Maekawa, Phys. Rev. Lett. 101, 247003 (2008).

[48] D.K.G. de Boer, C. Haas, G.A. Sawatzky, Phys. Rev. B 29, 4401 (1984); J. van den Brink, M.B.J. Meinders, J. Lorenzana, R. Eder, G.A. Sawatzky, Phys. Rev. Lett. 75, 4658 (1995). 
[49] J. Krzyszczak, T. Domański, K.I. Wysokiński, Acta Phys. Pol. A 118, 360 (2010); J. Krzyszczak, T. Domański, K.I. Wysokiński, R. Micnas, S. Robaszkiewicz, J. Phys. Condens. Matter 22, 255702 (2010); W. Chen, G. Khaliullin, O.P. Sushkov, Phys. Rev. B 83, 064514 (2011).

[50] A.M. Oleś, Phys. Rev. B 28, 327 (1983).

[51] P. Horsch, G. Khaliullin, A.M. Oleś, Phys. Rev. B 70 195103 (2004); A.M. Oleś, P. Horsch, G. Khaliullin, L.F. Feiner, Phys. Rev. B 72, 214431 (2005); N.N. Kovaleva, A.M. Oleś, A.M. Balbashov, A. Maljuk, D.N. Argyriou, G. Khaliullin, B. Keimer, Phys. Rev. B 81, 235130 (2010).

[52] L.F. Feiner, A.M. Oleś, Phys. Rev. B 59, 3295 (1999).

[53] A.M. Oleś, L.F. Feiner, Phys. Rev. B 65, 052414 (2002); L.F. Feiner, A.M. Oleś, Phys. Rev. B 71, 144422 (2005).

[54] M. Daghofer, A.M. Oleś, D.R. Neuber, W. von der Linden, Phys. Rev. B 73, 104451 (2006); M. Daghofer, A.M. Oleś, Acta Phys. Pol. A 111, 497 (2007).

[55] J.M. Tranquada, D.J. Buttrey, V. Sachan, J.E. Lorenzo, Phys. Rev. Lett. 73, 1003 (1994).

[56] S. Wakimoto, H. Kimura, K. Ishii, K. Ikeuchi, T. Adachi, M. Fujita, K. Kakurai, Y. Koike, J. Mizuki, Y. Noda, K. Yamada, A.H. Said, Y. Shvyd'ko, Phys. Rev. Lett. 102, 157001 (2009).

[57] M. Raczkowski, R. Frésard, A.M. Oleś, Phys. Rev. B 73, 094429 (2006)

[58] U. Schwingenschlögl, C. Schuster, R. Frésard, Europhys. Lett. 81, 27002 (2008).

[59] K. Rościszewski, A.M. Oleś, J. Phys. Condens. Matter 23, 265601 (2011).

[60] M. Daghofer, K. Wohlfeld, A.M. Oleś, E. Arrigoni, P. Horsch, Phys. Rev. Lett. 100, 066403 (2008); K. Wohlfeld, M. Daghofer, A.M. Oleś, P. Horsch, Phys. Rev. B 78, 214423 (2008); K. Wohlfeld, A.M. Oleś, M. Daghofer, P. Horsch, Acta Phys. Pol. A 115, 110 (2009).

[61] A.L. Chernyshev, A.H. Castro Neto, A.R. Bishop, Phys. Rev. Lett. 84, 4922 (2000); A.H. Castro Neto, S.R. White, A.L. Chernyshev, Phys. Rev. B 65, 214527 (2002); J.A. Riera, Phys. Rev. B 64, 104520 (2001).

[62] R. Eder, Y. Ohta, Phys. Rev. B 69, 094433 (2004); M. Vojta, T. Ulbricht, Phys. Rev. Lett. 93, 127002 (2004); P. Wróbel, A. Maciag, R. Eder, J. Phys. Condens. Matter 18, 1249 (2006).
[63] P. Wróbel, A.M. Oleś, Phys. Rev. Lett. 104, 206401 (2010).

[64] M. Daghofer, W. von der Linden, A.M. Oleś, Phys. Rev. B 70, 184430 (2004); A.M. Oleś, G. Khaliullin, Phys. Rev. B 85, 214414 (2011).

[65] J. Paglione, R.L. Greene, Nature Phys. 6, 645 (2010).

[66] M. Neupane, P. Richard, Y.-M. Xu, K. Nakayama, T. Sato, T. Takahashi, A.V. Federov, G. Xu, X. Dai, Z. Fang, Z. Wang, G.-F. Chen, N.-L. Wang, H.-H. Wen, H. Ding, Phys. Rev. B 83, 094522 (2011).

[67] L. Boeri, O.K. Andersen, Ann. Phys. 523, 8 (2011).

[68] G. Stollhoff, A.M. Oleś, V. Heine, Phys. Rev. B 41 7028 (1990); G. Stollhoff, A.M. Oleś, V. Heine, Phys. Rev. Lett. 76, 855 (1996).

[69] K. Haule, G. Kotliar, New J. Phys. 11, 025021 (2009).

[70] L. Boeri, O.V. Dolgov, A.A. Golubov, Phys. Rev Lett. 101, 026403 (2008).

[71] A. Ciechan, K.I. Wysokiński, Phys. Rev. B 80, 224523 (2009); A. Ciechan, K.I. Wysokiński, Acta Phys. Pol. A 118, 356 (2010).

[72] M. Daghofer, A. Moreo, J. Riera, E. Arrigoni, D.J. Scalapino, E. Dagotto, Phys. Rev. Lett. 101, 237004 (2008); A. Moreo, M. Daghofer, J.A. Riera, E. Dagotto, Phys. Rev. B 79, 134502 (2009).

[73] S. Raghu, X.-L. Qi, C.-X. Liu, D.J. Scalapino, S.-C. Zhang, Phys. Rev. B 77, 220503 (2008).

[74] Q. Si, E. Abrahams, Phys. Rev. Lett. 101, 076401 (2008).

[75] A. Nicholson, W. Ge, X. Zhang, J. Riera, M. Daghofer, A.M. Oleś, G. Martins, A. Moreo, E. Dagotto, Phys. Rev. Lett. 106, 217002 (2011).

[76] S. Graser, T.A. Maier, P.J. Hirschfeld, D.J. Scalapino, New J. Phys. 11, 025016 (2009).

[77] M. Daghofer, A. Nicholson, A. Moreo, E. Dagotto, Phys. Rev. B 81, 014511 (2010). 